\section{Avaliação da concordância de dados clínicos e demográficos entre Autorizações de Procedimento de Alta Complexidade Oncológica e prontuários de mulheres atendidas pelo Sistema Único de Saúde no Estado do Rio de Janeiro, Brasil}

\author{
Clinical and demographic data concordance \\ comparing Authorizations for High-Complexity \\ Oncological Procedures and patient records \\ of women treated under the Unified National \\ Health System in Rio de Janeiro, Brazil
}

1 Centro de Desenvolvimento Científico e Tecnológico, Secretaria de Estado de Saúde do Rio de Janeiro, Rio de Janeiro, Brasil.

2 Escola Nacional de Saúde Pública Sergio Arouca, Fundação Oswaldo Cruz, Rio de Janeiro, Brasil. 3 Escola Nacional de Ciências Estatísticas, Instituto Brasileiro de Geografia e Estatística Rio de Janeiro, Brasil.

Correspondência

C. Brito

Departamento de Administração

e Planejamento em Saúde, Escola Nacional de Saúde Pública Sergio Arouca, Fundação Oswaldo Cruz. Rua Leopoldo Bulhões 1480, Rio de Janeiro, $R J$ 21041-210, Brasil. cbrito@ensp.fiocruz.br

\section{Abstract}

This study investigates the concordance of clinical and demographic data under the Unified National Health System (SUS) in Brazil, comparing authorizations for high-complexity oncological procedures in breast cancer with a random sample of 310 corresponding medical records in Rio de Janeiro State, Brazil. Concordance was verified using kappa (K), prevalence-adjusted bias-adjusted kappa (PABAK), weighted-kappa (KW), intraclass correlation (ICC), and relative frequency statistics. 95\% confidence intervals were estimated, and Landis and Koch criteria were applied for concordance interpretation. Assuming PABAK as the most appropriate statistic for the variables analyzed, we observed a moderate concordance for cancer staging, $P A B A K=0.63$ (95\%CI: 0.56 0.70 ), and for morphological diagnosis, $P A B A K=$ 0.84 (95\%CI: 0.72-0.96). For date of birth and date of diagnosis, ICCs were 0.96 (95\%CI: 0.95-0.96) and 0.92 (95\%CI: 0.90-0.94), respectively. Concordance regarding municipality of residence was 52.9\%. The study indicates that, except for municipality of residence, the data concordance comparing authorizations for procedures and patient medical records in Rio de Janeiro allows its application to epidemiological and health services research, as well as for health planning purposes.

Women's Health; Breast Cancer; Reliability and Validity
Cláudia Brito 1,2

Margareth Crisóstomo Portela 2

Mauricio Teixeira Leite de Vasconcellos 3

\section{Introdução}

No final da última década, substanciais modificações foram implementadas na forma de credenciamento de serviços oncológicos no âmbito do Sistema Único de Saúde (SUS). Até outubro de 1998, não era possível identificar os tipos de tumores nem os pacientes que eram tratados pelo SUS, além de ser impossível identificar qual era a finalidade terapêutica dos procedimentos executados. Em setembro de 1998, com a publicação da Portaria GM n. 3.535 1, o Ministério da Saúde do Brasil (MS) conduziu as transformações regulamentares na área da assistência oncológica. Essas mudanças objetivaram a prestação de atendimento integral aos pacientes oncológicos e a articulação de todas as etapas do tratamento do câncer. Assim, o MS estabelece que, para credenciamento oncológico com o SUS, as unidades solicitantes tenham de ter na sua estrutura condições de atender a todas as necessidades básicas para o tratamento das neoplasias mais freqüentes no país.

Concomitante com a Portaria GM n. 3.535 1, que estabeleceu uma rede hierarquizada para atendimento oncológico e os critérios para cadastramento do Centro de Alta Complexidade em Oncologia (CACON), houve a necessidade de individualizar e ampliar as informações acerca dos tumores e dos pacientes oncológicos, controlando os procedimentos executados por meio de autorização prévia regulamentada e 
informada em um sistema de informações mais detalhado. Para tanto, o MS publicou a Portaria GM n. $3.536^{2}$, que determinava a implantação do Sistema de Autorização de Procedimentos de Alta Complexidade na Área Oncológica (SIA/APAC-ONCO).

O SIA/APAC-ONCO, apesar de ter sido criado e desenvolvido com uma lógica contábil de controle para pagamento de procedimentos oncológicos, representa um avanço em relação aos demais sistemas de informações para pagamento de procedimentos do SUS (SIH e SIA). As Autorizações de Procedimento de Alta Complexidade Oncológica (APAC-ONCO) contemplam informações que permitem a identificação dos pacientes, o conhecimento de informações clínicas essenciais para o uso em pesquisas, e a descrição dos procedimentos quimioterápicos que passou a ser baseada nas denominações tumorais e nas finalidades terapêuticas. As alterações no sistema de informações do SUS vêm possibilitando maior diversidade de ações que visam à melhoria da qualidade dos serviços de saúde, por meio do provimento de informações para a tomada de decisão mais racional pelos dirigentes, para execução de ações de controle e avaliação da qualidade dos serviços e para realização de pesquisas científicas. Nesse sentido, o sistema de informação tornou-se um instrumento estratégico para a melhoria da gestão.

A grande vantagem do uso de bancos de dados administrativos em pesquisas e na tomada de decisão pelos gestores de saúde está relacionada à sua rápida disponibilidade e, conseqüentemente, a economia de tempo e de recursos 3 . Entretanto, sem o conhecimento da qualidade das informações utilizadas não se sabe se os resultados estão isentos de interferências que implicam distorções nas conclusões obtidas. Esse aspecto é crítico para a utilização de dados administrativos em qualquer pesquisa e na tomada de decisão gerencial.

Coutinho et al. 4 descrevem, por exemplo, a importância da avaliação da confiabilidade nos estudos epidemiológicos, visto que os erros de classificação (viés de informação) da variável de exposição de um estudo produzem distorções na estimativa de riscos, que subestimam ou superestimam o efeito da exposição. Confiabilidade é o termo utilizado para dimensionar a reprodutibilidade dos resultados, obtida por diferentes pessoas ou em diferentes momentos 5,6.

Apesar de as APAC-ONCO representarem uma importante fonte de dados para pesquisa, planejamento e avaliação em saúde na área oncológica, com crescente utilização por parte de dirigentes, gestores e pesquisadores $7,8,9,10$, não foram encontrados estudos que tenham testado a sua confiabilidade.

Assim, diante da importância de ações de controle do câncer de mama e do potencial de utilização de um banco de dados nacional sobre informações oncológicas, foi realizado este estudo com o propósito de avaliar a confiabilidade das informações clínicas e demográficas contidas nas APAC-ONCO de câncer de mama, no Estado do Rio de Janeiro.

\section{Métodos}

A população alvo deste estudo é constituída por pacientes com câncer de mama feminina que realizaram quimioterapia pelo SUS, em pelo menos uma das 15 unidades credenciadas pelo SUS no Estado do Rio de Janeiro, entre novembro de 1999 e novembro de 2002. Assim, foi elaborado um cadastro contendo todas as pacientes com câncer de mama que realizaram quimioterapia por intermédio do SUS e, portanto, que foram cadastradas no SIA/APAC-ONCO no período de interesse. Para tanto, foram excluídos do cadastro de seleção pacientes do sexo masculino, pacientes com código de procedimento não-compatível com o diagnóstico de câncer de mama e unidades não-credenciadas no período de estudo. As mulheres que, no período referido, foram tratadas em mais de uma unidade do estudo foram associadas a uma única unidade oncológica credenciada: a unidade onde receberam atendimento por maior número de meses. Esse procedimento objetivou não alterar as probabilidades de seleção da amostra pela inclusão de uma mesma paciente nas diferentes unidades onde buscou tratamento. Com esses procedimentos foi gerado um cadastro de seleção de 10.862 pacientes, cuja distribuição consta da Tabela 1, com base no qual foi selecionado uma amostra aleatória simples de 310 prontuários desenhada para estimar a idade média das pacientes com erro relativo de 2,5\% e nível de significância de 5,0\%. Para garantir representatividade dos diversos segmentos da população, a seleção da amostra foi sistemática com os registros do cadastro ordenados por grupo (CACON, isolada), unidade e classe de idade no diagnóstico. As informações contidas nos prontuários da amostra foram usadas para testar a concordância dos dados do cadastro APAC-ONCO de quimioterapia nos casos de câncer de mama feminina.

Não houve substituição de pacientes por perda de prontuários. Entretanto, houve três casos de substituição, um no Instituto Nacional de Câncer (INCA) - erro no cadastro, pacien- 
Distribuição da amostra por unidade credenciada.

\begin{tabular}{|c|c|c|c|}
\hline Município & Nome fantasia & $\begin{array}{l}\text { Pacientes } \\
\text { no cadastro }\end{array}$ & $\begin{array}{l}\text { Tamanho } \\
\text { da amostra }\end{array}$ \\
\hline Campos & Clínica Santa Maria Ltda. & 439 & 12 \\
\hline Campos & Instituto Endocrinologia e Medicina Nuclear & 182 & 5 \\
\hline Itaperuna & Conferência São José do Avaí & 117 & 4 \\
\hline Magé & Med Center Magé & 30 & 1 \\
\hline Mesquita & Hospital da UNIG & 162 & 5 \\
\hline Niterói & $\begin{array}{l}\text { Hospital Universitário Antonio Pedro } \\
\text { (Universidade Federal Fluminense) }\end{array}$ & 31 & 1 \\
\hline Nova Iguaçu & Instituto Oncológico & 396 & 11 \\
\hline Petrópolis & Centro de Terapia Oncológica & 462 & 13 \\
\hline Rio de Janeiro & Instituto Nacional de Câncer & 7.318 & 209 \\
\hline Rio de Janeiro & Hospital Mario Kroeff & 541 & 15 \\
\hline Rio de Janeiro & $\begin{array}{l}\text { Hospital Universitário Pedro Ernesto } \\
\text { (Universidade do Estado do Rio de Janeiro) }\end{array}$ & 99 & 3 \\
\hline Rio de Janeiro & $\begin{array}{l}\text { Hospital Universitário Clementino Fraga Filho } \\
\text { (Universidade Federal do Rio de Janeiro) }\end{array}$ & 459 & 13 \\
\hline Rio de Janeiro & $\begin{array}{l}\text { Hospital Universitário Gaffrée \& Guinle } \\
\text { (Universidade Federal do Estado do Rio de Janeiro) }\end{array}$ & 81 & 2 \\
\hline Teresópolis & Hospital São José & 111 & 3 \\
\hline Volta Redonda & Radiclin & 434 & 13 \\
\hline Total & & 10.862 & 310 \\
\hline
\end{tabular}

Fonte: SIA/APAC-Quimioterapia.

te do sexo masculino -, e os outros dois, um na Clínica Santa Maria e outro na Med Center Magé, foram decorrentes do fato de não constar qualquer informação que comprovasse atendimento nas respectivas unidades no prontuário encontrado, apesar do banco de dados que gerou a amostra ter sido proveniente de faturamento no SUS. As substituições foram feitas respeitando o desenho da amostra, ou seja, foi selecionada a paciente (ou o prontuário) seguinte na ordem de seleção.

A rigor, o desenho usado nesta pesquisa não permite avaliar a validade das variáveis investigadas, pois os dados (prontuários e APAC) utilizados são secundários. Assim, este estudo centra-se na avaliação da concordância das informações das APAC com as dos prontuários.

As variáveis que tiveram a sua confiabilidade analisada foram o estadiamento, o diagnóstico morfológico, a data de nascimento, a data de diagnóstico e o município de residência. $\mathrm{O}$ número de casos usados na comparação dos resultados entre a APAC-ONCO e os prontuários foi distinto por variável, em decorrência da variação na freqüência de informações válidas encontradas nos prontuários.

Foram utilizados diferentes métodos para avaliar a concordância dos valores, uma vez que as variáveis são de diferentes tipos (categorizadas ou contínuas), sendo aplicado o método mais adequado para cada tipo de variável. Assim, para medir a confiabilidade das variáveis categóricas, estadiamento e diagnóstico morfológico, foi utilizado a estatística kappa (K), devido à proporção de concordância calculada por este método excluir as concordâncias ao acaso. Quanto ao estadiamento, variável do tipo ordinal, foi também calculado o kappa ponderado $(\mathrm{KW})$, com ponderação linear, em razão de esta abordagem atribuir uma ponderação à discordância e fornecer uma estimativa adequada do coeficiente de confiabilidade.

Em alguns estudos de concordância, estimar uma única medida sumária de concordância é suficiente. Porém, para Silva \& Pereira 11, em contextos mais complexos, a estatística kappa apresenta características indesejáveis. A prevalência de uma dada condição na população pode afetar o resultado final, e baixas prevalências tendem a estar associadas a baixos níveis de reprodutibilidade 12 . Uma vez que o valor de kappa depende da concordância devido ao acaso, é possível encontrar-se pequenos valores de kappa devido à prevalência do evento e não a erros substanciais relacionados ao procedimento de aferição empregado 13. 
Dessa forma, para o estadiamento e para o diagnóstico morfológico, estimou-se, também, o kappa ajustado pela prevalência (KA) comparando-o com o kappa. Para a obtenção dessas estatísticas utilizou-se o PEPI, versão 3.

A confiabilidade das variáveis contínuas, data de nascimento e data de diagnóstico, foi medida utilizando-se o coeficiente de correlação intraclasse (CCIC).

Para todas as estatísticas foram estimados os intervalos de confiança de $95,0 \%(\mu=0,05)$. Usou-se os critérios de Landis \& Koch 13 para interpretação da concordância, assim definidos: (a) quase-perfeita, para valores de 0,81 a 1,00; (b) substancial, para valores de 0,61 a 0,80 ; (c) moderada, para valores entre 0,41 e 0,60 ; (d) regular, para valores entre 0,21 e 0,40 ; (d) discreta, para valores de 0 a 0,20 ; e (e) pobre, para valores negativos.

Para verificar a concordância da variável município de residência utilizou-se a concordância observada, mesmo sabendo que este método resulta em valores um pouco superio- res ao da estatística kappa. No entanto, não foi possível estimar o kappa devido ao grande número de combinações encontradas nos valores dessa variável.

\section{Resultados}

A Tabela 2 apresenta a comparação das informações amostrais obtidas nos prontuários e os dados correspondentes do cadastro de seleção para as variáveis estadiamento e diagnóstico morfológico. Observa-se que a ordem de prevalência dos quatro diferentes estádios é a mesma entre as duas fontes de dados. Em relação ao diagnóstico morfológico, apesar de os diagnósticos menos prevalentes terem tido uma alta variação entre fonte de dados, os diagnósticos mais prevalentes seguiram a mesma ordenação, independente da pequena diferença de valor entre eles. Assim, o carcinoma ductal infiltrante (M85003) representou 85,48\% segun-

Tabela 2

Distribuição amostral das variáveis estadiamento e CID-morfológico nos prontuários e na APAC.

\begin{tabular}{|c|c|c|c|c|}
\hline \multirow[t]{2}{*}{ Variável } & \multicolumn{2}{|c|}{ Prontuários } & \multicolumn{2}{|c|}{ APAC } \\
\hline & $\mathrm{n}$ & $\%$ & $\mathrm{n}$ & $\%$ \\
\hline Estadiamento & 310 & 100,00 & 310 & 100,00 \\
\hline I & 27 & 8,71 & 20 & 6,45 \\
\hline II & 123 & 39,68 & 122 & 39,35 \\
\hline III & 98 & 31,61 & 102 & 32,90 \\
\hline IV & 38 & 12,26 & 66 & 21,29 \\
\hline Ignorado & 24 & 7,74 & & \\
\hline CID morfológico & 310 & 100,00 & 310 & 100,00 \\
\hline M80000 - Neoplasia benigna & 0 & 0,00 & 13 & 4,19 \\
\hline M80003 - Neoplasia maligna & 0 & 0,00 & 1 & 0,32 \\
\hline M80103 - Carcinoma SOE & 0 & 0,00 & 8 & 2,58 \\
\hline M80213 - Carcinoma anaplásico SOE & 1 & 0,32 & 0 & 0,00 \\
\hline M80223 - Carcinoma pleomórfico & 0 & 0,00 & 1 & 0,32 \\
\hline M80503 - Carcinoma papilar SOE & 7 & 2,26 & 2 & 0,65 \\
\hline M81403 - Adenocarcinoma SOE & 0 & 0,00 & 2 & 0,65 \\
\hline M84803 - Adenocarcinoma mucinoso & 4 & 1,29 & 3 & 0,97 \\
\hline M85002 - Carcinoma intraductal não infiltrante SOE & 1 & 0,32 & 0 & 0,00 \\
\hline M85003 - Carcinoma de dutos infiltrante (C50.-) & 265 & 85,48 & 260 & 83,87 \\
\hline M85013 - Comedocarcinoma SOE (C50.-) & 1 & 0,32 & 1 & 0,32 \\
\hline M85103 - Carcinoma medular SOE & 2 & 0,65 & 2 & 0,65 \\
\hline M85203 - Carcinoma lobular SOE (C50.-) & 18 & 5,81 & 7 & 2,26 \\
\hline M85213 - Carcinoma ductular infiltrante (C50.-) & 0 & 0,00 & 5 & 1,61 \\
\hline M85223 - Carcinoma infiltrante de dutos e lobular (C50.-) & 0 & 0,00 & 5 & 1,61 \\
\hline M89103 - Rabdomiossarcoma embrionário & 1 & 0,32 & 0 & 0,00 \\
\hline Ignorado & 10 & 3,23 & & \\
\hline
\end{tabular}


do os prontuários analisados e $83,87 \%$ de acordo com a APAC. O segundo tumor mais prevalente foi o carcinoma lobular SOE (M85203) para ambas as fontes, representando $5,81 \%$ da população estudada segundo os prontuários, e $2,26 \%$, segundo a APAC. Essa seqüência de prevalências de tipo de tumor da mama segue o encontrado na população em geral 14 .

A Tabela 3 apresenta o resultado dos diversos procedimentos utilizados para aferir a confiabilidade dos distintos tipos de variáveis. $\mathrm{Pa}-$ ra a variável estadiamento, o kappa indicou uma confiabilidade moderada de 0,60 (IC95\%: 0,52-0,67). O kappa ajustado pela prevalência foi classificado como substancial 12 e superior ao kappa simples, atingindo 0,63 (IC95\%: 0,560,70 ) de concordância. Isso decorre da prevalência de estadiamento II na população estudada. O kappa ponderado, que atribui peso diferente às discordâncias observadas, teve um índice de confiabilidade de 0,64 (IC95\%: 0,57$0,71)$. A concordância observada, por não excluir a concordância decorrente do acaso, teve um valor maior do que o kappa, apresentando $66,78 \%$ de confiabilidade (Tabela 3 ).

Em relação às categorias de estadiamento, o estádio III teve uma concordância substancial ( $K=0,62$; IC95\%: 0,52-0,72). Os estádios I, II e IV tiveram uma concordância moderada com os valores de 0,51 (IC95\%: 0,32-0,70), 0,60 (IC95\%: 0,51-0,70) e 0,59 (IC95\%: 0,47-0,72).

A Tabela 3 mostra, para a variável diagnóstico morfológico, uma confiabilidade regular de 0,37 (IC95\%: 0,25-0,50). Entretanto, para o kappa ajustado pela prevalência, indica uma concordância quase perfeita $(0,84)$. Esse fato pode ser explicado pela alta prevalência, na população estudada, do diagnóstico de carcinoma ductal infiltrante, o que afetou o valor final do kappa.

Observa-se, ainda na Tabela 3, a confiabilidade das variáveis data de nascimento e data de diagnóstico, com estimativas do CCIC para data de nascimento e data de diagnóstico de 0,96 (IC95\%: 0,95-0,96) e 0,92 (IC95\%: 0,90290,9381), respectivamente. Ambos considerados quase-perfeitos segundo critérios de Landis \& Koch 13. Em relação à variável município de residência, os dados revelaram um baixo porcentual de concordância, apenas 52,90\%.

\section{Discussão}

Com base nos resultados deste estudo, observou-se que a qualidade dos dados clínicos e demográficos da APAC de câncer de mama no Estado do Rio de Janeiro foi bastante satisfató-

Tabela 3

Estimativas de concordância entre APAC-ONCO de quimioterapia e prontuários por estatística de concordância, segundo as variáveis avaliadas.

\begin{tabular}{|c|c|c|c|c|c|c|}
\hline \multirow[t]{2}{*}{ Variáveis avaliadas } & \multirow[t]{2}{*}{$\mathbf{n}$} & \multicolumn{5}{|c|}{ Estatística de concordância } \\
\hline & & kappa (IC95\%) & KW (IC95\%) & KA (IC95\%) & $\mathrm{CCIC} \mathrm{(IC95 \% )}$ & $\%$ \\
\hline \multirow[t]{2}{*}{ Estadiamento } & 286 & 0,60 & 0,64 & 0,63 & & 66,78 \\
\hline & & $(0,52-0,67)$ & $(0,57-0,71)$ & $(0,56-0,70)$ & & \\
\hline \multirow[t]{2}{*}{1} & & 0,51 & & & & \\
\hline & & $(0,32-0,70)$ & & & & \\
\hline \multirow[t]{2}{*}{ II } & & 0,60 & & & & \\
\hline & & $(0,51-0,70)$ & & & & \\
\hline \multirow[t]{2}{*}{ III } & & 0,62 & & & & \\
\hline & & $(0,52-0,72)$ & & & & \\
\hline \multirow[t]{2}{*}{ IV } & & 0,59 & & & & \\
\hline & & $(0,47-0,72)$ & & & & \\
\hline \multirow[t]{2}{*}{ CID morfológico } & 300 & 0,37 & & 0,84 & & \\
\hline & & $(0,25-0,50)$ & & $(0,72-0,96)$ & & \\
\hline \multirow[t]{2}{*}{ Data de nascimento } & 310 & & & & 0,96 & 92,58 \\
\hline & & & & & $(0,95-0,97)$ & \\
\hline \multirow[t]{2}{*}{ Data de diagnóstico } & 310 & & & & 0,92 & \\
\hline & & & & & $(0,90-0,94)$ & \\
\hline Município de residência & 310 & & & & & 52,90 \\
\hline
\end{tabular}

$\mathrm{KW}=$ kappa ponderado; $\mathrm{KA}=$ kappa ajustado; $\mathrm{CCIC}=$ coeficiente de correlação intraclasse. 
ria. A boa concordância das variáveis investigadas, que são as consideradas como sendo os principais fatores prognósticos para o câncer de mama, depõe favoravelmente para a utilização desses registros administrativos em pesquisas epidemiológicas e de avaliação da qualidade em relação a essa neoplasia. É certo que outros fatores prognósticos importantes não puderam ser analisados por não estarem disponíveis na APAC-ONCO de quimioterapia. A grande limitação esteve relacionada à precariedade da confiabilidade da variável município de residência.

O baixo valor de concordância da variável município de residência deve estar mais relacionado ao erro de informação na APAC-ONCO para as residentes fora da capital do que a erro de informação das pacientes. Essa conclusão decorre da existência, nos prontuários, de cópias de comprovantes de residência e etiquetas de identificação com indicação do Município do Rio de Janeiro. Curiosamente, o campo bairro, da etiqueta, indicava o município correto.

A informação acerca da procedência dos pacientes atendidos pelos diversos serviços credenciados é considerada essencial sob o ponto de vista do planejamento do sistema, da programação dos serviços, da alocação dos recursos financeiros segundo as diferentes necessidades e do conhecimento da "real" rede de referência. Entretanto, essa informação, tomando como base a amostra pesquisada, foi considerada de baixa qualidade na APAC.

O SIA/APAC-ONCO, talvez por ter sido implantado mais recentemente, é considerado mais completo que o SIA/SUS de média complexidade. Entretanto, várias informações que constam como preenchimento obrigatório nas APAC não são digitadas no sistema informatizado. Isto representa trabalho inútil, diminui a possibilidade de desenvolvimento de pesquisas e gera a necessidade de recorrer a outras fontes de dados complementares.

Outro problema que merece destaque refere-se à estruturação do SIA/APAC-ONCO que não possibilita facilmente o conhecimento do número de pacientes novos e, conseqüentemente, da taxa de incidência e/ou captação por cada serviço e do medicamento utilizado no tratamento do câncer. Esses fatos não só afetam o desenvolvimento de pesquisas nessa área, pois dificultam a comparação dos dados, como também a atuação do controle e avaliação dos serviços pelos órgãos responsáveis pela gestão do SUS.

A boa confiabilidade das informações analisadas na APAC, acrescida da vantagem desse banco estar implantado, disponível e sempre atualizado, visto ser fonte de receita das unidades, torna-o uma valiosa ferramenta de apoio a inúmeras ações cotidianas dos gestores do sistema de saúde.

As evidências obtidas permitem concluir que, com exceção da variável município de residência, as informações analisadas mostraram concordância com as dos prontuários, abrindo espaço para o uso da APAC-ONCO em estudos epidemiológicos e no planejamento em saúde, tendo em vista que seu uso representa uma economia substancial de tempo e recursos, quando comparado com a alternativa de uso dos prontuários. Entretanto, estudos que requeiram maior precisão sobre informações clínicas inevitavelmente deverão ser baseados em prontuários.

Por fim, apesar de esse banco de dados não ter sido concebido para usos em pesquisas e na tomada de decisão gerencial, parece necessário que haja investimento para que os sistemas de informações relativos à saúde sejam integrados e que o processo de reformulação desses sistemas seja conduzido de forma mais participativa, visando à ampliação de seus usos. A integração das informações de saúde, atualmente dispersas em vários sistemas, é um imperativo para a eficiência da aplicação dos recursos públicos, sobretudo para um país da dimensão do Brasil, em uma realidade de recursos escassos. 


\section{Resumo}

Este estudo buscou avaliar a concordância dos dados clínicos e demográficos das Autorizações de Procedimentos de Alta Complexidade (APAC) em câncer de mama, do Sistema Único de Saúde (SUS), com os encontrados em uma amostra aleatória de 310 prontuários, no Estado do Rio de Janeiro, Brasil. A concordância foi calculada pelas estatísticas kappa (K), kappa ajustado pela prevalência (KA), kappa ponderado (KW), coeficiente de correlação intraclasse (CCIC) e freqüência relativa. Foram estimados intervalos de confiança (IC) de 95,0\% e usado o critério de Landis \& Koch para interpretação da concordância. Assumindo KA como a estatística mais apropriada às características das variáveis analisadas, observou-se uma boa concordân-

\section{Colaboradores}

Todos os autores participaram do planejamento do projeto de pesquisa, da análise dos dados e da elaboração do artigo. C. Brito coordenou a pesquisa de campo e realizou a coleta, digitação e crítica dos dados.

\section{Referências}

1. Brasil. Portaria GM n. 3.535. Estabelece uma rede hierarquizada dos Centros que prestam assistência oncológica e atualiza os critérios mínimos para o cadastramento de Centros de Alta Complexidade em Oncologia. Diário Oficial da União 1998; 2 set.

2. Brasil. Portaria GM n. 3.536. Determina a implantação do Sistema de Autorização de Procedimentos de Alta Complexidade na área de oncologia (APAC/ONCO). Diário Oficial da União 1998; 2 set.

3. Escosteguy CC, Portela MC, Medronho RA, Vasconcellos MTL. O Sistema de Informações Hospitalares e a assistência ao infarto agudo do miocárdio. Rev Saúde Pública 2002; 36:491-9.

4. Coutinho ESF, Silva SD, Paiva CHA, Freitas GG, Miranda LP, Mendonça R. Confiabilidade da informação sobre uso recente de medicamentos em um estudo caso-controle de base hospitalar. Cad Saúde Pública 1999; 15:553-8.

5. Almeida Filho N. Epidemiologia sem números: uma introdução crítica à ciência epidemiológica. Rio de Janeiro: Campus; 1989.

6. Armstrong BK, White E, Saracci R. Principles of exposure measurement in epidemiology. New York: Oxford University Press; 1994.

7. Bertholasce ACA, Brito C. Diretrizes para reorganização, reorientação e acompanhamento da assistência oncológica no Estado do Rio de Janeiro. http://www.saude.rj.gov.br/gestor/relonco.shtml (acessado em 12/Jan/2004).

8. Brito C. Avaliação do tratamento à paciente com câncer de mama nas unidades oncológicas do Sistema Único de Saúde no Estado do Rio de Ja- cia para estadiamento da doença, KA = 0,63 (IC95\%: 0,56-0,70) e para diagnóstico morfológico, $K A=0,84$ (IC95\%: 0,72-0,96). O CCIC estimado para data de nascimento e data de diagnóstico foi de 0,96 (IC95\%: 0,95-0,96) e 0,92 (IC95\%: 0,90-0,94), respectivamente. Em relação ao município de residência, a concordância observada foi de 52,9\%. Exceto pelo município de residência, as informações analisadas mostraram concordância com as dos prontuários, abrindo espaço para o seu uso em estudos epidemiológicos e serviços de saúde, e no planejamento em saúde.

Saúde da Mulher; Câncer da Mama; Confiabilidade e Validade

\section{Agradecimentos}

Os autores agradecem a Paulo Roberto Borges de Souza Júnior pelo auxílio nos cálculos dos testes de confiabilidade; a José Luiz Zedane e Vinícius Braga di Salvo pela colaboração na elaboração do banco de dados; e à Dra. Claudia Caminha Escosteguy e ao Dr. Sérgio Koifman pelas sugestões dadas.

neiro [Dissertação de Mestrado]. Rio de Janeiro: Escola Nacional de Saúde Pública, Fundação Oswaldo Cruz; 2004.

9. Teixeira OGS, Faria HP, Campos FE, Almeida MCM. Acesso aos procedimentos de alta complexidade no âmbito do SUS em Belo Horizonte/ MG: o caso da utilização da quimioterapia e radioterapia por pacientes portadores de câncer de mama residentes em Belo Horizonte, nos anos de 2000 e 2001. In: Anais do VI Congresso Brasileiro de Epidemiologia [CD-ROM]. Recife: ABRASCO; 2004.

10. Cardoso VS, Costa MCE, Sharapim MP, Pinheiro R, Oliveira MR. Uso de sistemas de Informação do SUS na avaliação da atenção ao câncer de mama na cidade do Rio de Janeiro. In: Anais do VI Congresso Brasileiro de Epidemiologia [CD-ROM]. Recife: ABRASCO; 2004.

11. Silva EF, Pereira MG. Avaliação das estruturas de concordância e discordância nos estudos de confiabilidade. Rev Saúde Pública 1998; 32:383-93.

12. Byrt T, Bishop J, Carlin JB. Bias, prevalence and kappa. J Clin Epidemiol 1993; 46:423-9.

13. Landis JR, Kock GG. The measurement of observer agreement for categorical data. Biometrics 1977; 33:159-75.

14. Eisenberg ALA, Koifman S. Aspectos gerais dos adenocarcinomas de mama, estadiamento e classificação histopatológica com descrição dos principais tipos. Rev Bras Cancerol 2000; 46:265-74.

Recebido em 18/Ago/2004

Versão final reapresentada em 20/Abr/2005 Aprovado em 31/Mai/2005 